\title{
Pulmonary Nodular Amyloidosis
}

\section{Dr Usman Sheikh, MBBS, MRCP; Dr Matthew Brown, FRCPath; Dr Alasdair Taylor, FRCR Department of Radiology \& Diagnostic Imaging and Department of Pathology, RLI}

\section{CLINICAL PRESENTATION}

62 year old lady, heavy smoker and known case of chronic obstructive pulmonary disease (COPD) presented with history of cough, weight loss, night sweats and increasing tiredness. She also complained of left shoulder pain and breathlessness on exertion.

Chest x-ray showed left upper lobe mass lesion.

A CT Thorax was arranged for further evaluation and showed a $1.3 \mathrm{~cm} \times 1.1 \mathrm{~cm}$ spiculated mass in left upper lobe and also $1 \mathrm{~cm}$ nodule in the left lower lobe.

A PET-FDG was arranged which concluded that the suspect lung nodules were not metabolically active and therefore probably benign/ post inflammatory, but as a small proportion of adenocarcinoma will show limited or no FDG expression, these will require review CT to asses growth rate as per local lung nodule protocol.

Follow-up CT scans showed further increases in the size of lesions and a CT guided biopsy was performed.

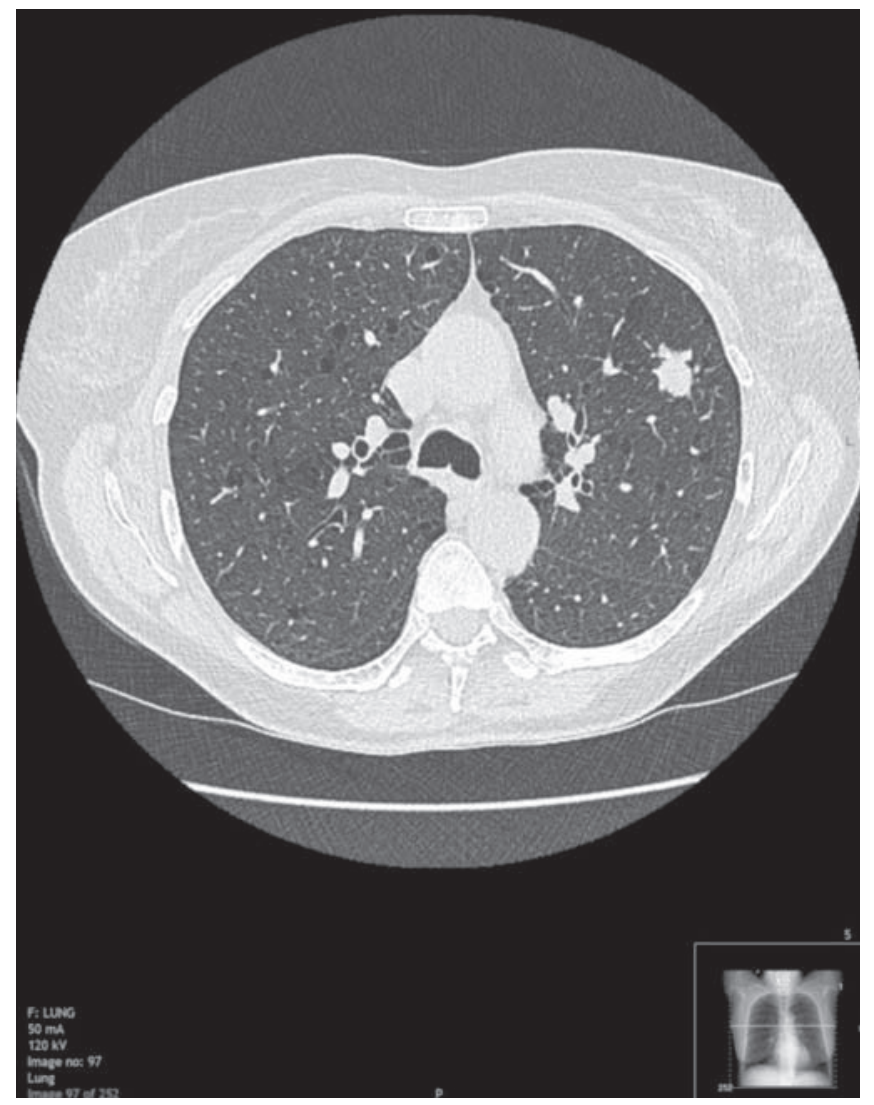

CT Thorax

\section{DIAGNOSIS}

A CT guided biopsy confirmed the diagnosis of pulmonary nodular amyloidosis.

\section{RADIOGRAPHIC FEATURES}

Characteristic features that have been described on CT are

- sharp, smooth and lobulated contours
- slow growth: often over years and with no regression

- variable shapes and sizes ranging from $0.5 \mathrm{~cm}-15 \mathrm{~cm}$

- calcification within the nodule in $50 \%$ cases with often central or irregular pattern

- associated cavitation - very rare

- lung cysts (rare) (described with localized amyloidosis in Sjögren syndrome)

\section{HISTOPATHOLOGICAL FEATURES}

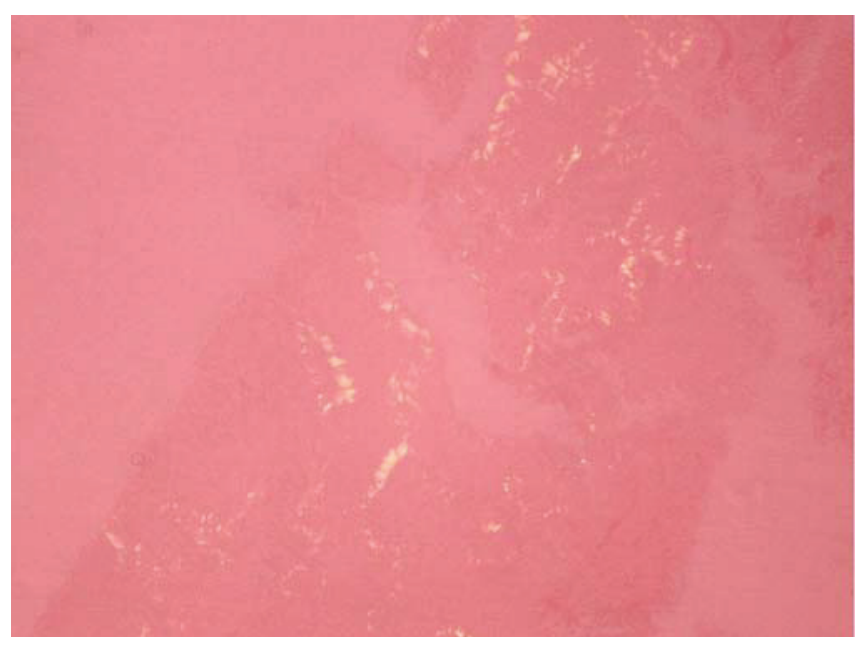

Glassy pink material on $\mathrm{H}+\mathrm{E}$ which appears red with Congo red staining and shows apple green birefringence on polarised light microscopy, as shown above. It is important to subtype the amyloid deposits in order to determine the underlying cause and guide any treatment. This can be done by a range of methods including electron microscopy and mass spectrometry but is now most commonly done by immunohistochemistry. AA and AL amyloid are the most common subtypes.

\section{PULMONARY NODULAR AMYLOIDOSIS}

Pulmonary amyloidosis is a localized process instead of systemic disease. Two patterns identified are nodular parenchymal and the alveolar septal. Both have nonspecific radiographic findings.

\section{Conflict of interest - none}

\section{REFERENCES}

Czeyda-Pommersheim F, Hwang M, Chen SS, et al. Amyloidosis: modern cross-sectional imaging [published online]. RadioGraphics. 2015;35(5) Available at: http:// pubs.rsna.org/doi/full/10.1148/rg.2015140179 (accessed 21.3.18)

Correspondence to: Usman A Sheikh, Specialist Registrar Radiology at RLI Usman.sheikh@mbht.nhs.uk 